\title{
Ultra-Wideband Range Estimation: Theoretical Limits and Practical Algorithms
}

\author{
Invited Paper \\ Ismail Guvenc*, Sinan Gezici ${ }^{\sharp}$, and Zafer Sahinoglu ${ }^{\natural}$ \\ * DoCoMo Communications Laboratories USA, Inc., 3240 Hillview Avenue, Palo Alto, CA 94304, USA \\ $\sharp$ Department of Electrical and Electronics Engineering, Bilkent University, Bilkent, Ankara 06800, Turkey \\ Ł Mitsubishi Electric Research Laboratories, 201 Broadway Avenue, Cambridge, MA 02139, USA \\ iguvenc@docomolabs-usa.com, gezici@ieee.org, zafer@merl.com
}

\begin{abstract}
The high time resolution of ultra-wideband (UWB) signals enables wireless devices to perform accurate range estimation. In order to realize UWB systems with accurate ranging capabilities, both theoretical limits on range estimation and practical algorithms that approach those limits should be investigated. This paper provides a survey of various UWB ranging algorithms and discusses their performance and complexity tradeoffs. In addition, theoretical limits on range estimation are discussed in terms of Cramer-Rao and Ziv-Zakai lower bounds.

Index Terms-Ultra-wideband (UWB), time-of-arrival (TOA) estimation, ranging, Cramer-Rao lower bound (CRLB), ZivZakai lower bound (ZZLB).
\end{abstract}

\section{INTRODUCTION}

Large bandwidths of ultra-wideband (UWB) signals make them a good candidate for applications that require high speed communications or accurate position estimation [1][3]. For positioning applications, large bandwidths of UWB signals provide high time resolution, which, in theory, can facilitate centimeter-accuracy range estimation. However, in practical systems, it can be difficult to achieve the theoretical limits of UWB range estimation due to constraints on power, computational complexity, and/or cost. Therefore, it is of great importance to evaluate the performance of practical UWB range estimation algorithms and compare them against theoretical limits.

This paper provides a survey on various UWB range estimation algorithms proposed in the literature, and compares them in terms of complexity and performance. In addition, theoretical lower bounds on range estimation are studied in terms of Cramer-Rao lower bounds (CRLBs) and Ziv-Zakai lower bounds (ZZLBs). The aim is to present fundamental limits on range estimation together with some practical range estimation algorithms, and thus to provide an intuition for the differences between the theoretical limits and the accuracies that can be achieved under practical constraints.

\section{Signal MODEL}

Consider an impulse radio (IR) UWB system with the following transmitted ranging signal [2]

$$
\tilde{s}(t)=\sqrt{E_{\mathrm{s}}} \sum_{j=-\infty}^{\infty} a_{j} \tilde{\omega}\left(t-j T_{\mathrm{f}}-c_{j} T_{\mathrm{c}}\right),
$$

where $E_{\mathrm{s}}$ represents the energy of a ranging symbol, $a_{j} \in$ $\{-1,+1\}$ is a polarity code, $\tilde{\omega}(t)$ denotes a UWB pulse, $T_{\mathrm{f}}$ is the frame duration, $c_{j} \in\left\{0,1, \ldots, N_{\mathrm{h}}-1\right\}$ is a time-hopping (TH) code, $T_{\mathrm{c}}$ is the chip duration, and $N_{\mathrm{h}}$ is the number of

\footnotetext{
${ }^{0}$ This research was supported in part by the European Commission in the framework of the FP7 Network of Excellence in Wireless COMmunications NEWCOM++ (contract no. 216715) and WiMAGIC (contract no. 215167).
}

chips per frame. It is assumed that the width of the UWB pulse $\tilde{\omega}(t), T_{\mathrm{p}}$, is smaller than $T_{\mathrm{c}}$.

In this signal model, each ranging symbol is modeled to consist of a number $N_{\mathrm{f}}$ of pulses, and each pulse resides in a frame interval $T_{\mathrm{f}}$. Therefore, the duration of a ranging symbol is given by $T_{\mathrm{s}}=N_{\mathrm{f}} T_{\mathrm{f}}$. If the ranging signal in (1) is transmitted over a multipath channel with $L$ multipath components, the received signal can be expressed as

$$
r(t)=\sum_{l=1}^{L} \alpha_{l} s\left(t-\tau_{l}\right)+n(t),
$$

where $\alpha_{l}$ and $\tau_{l}$ are, respectively, the channel coefficient and the delay of the $l$ th multipath component, $n(t)$ is zeromean white Gaussian noise with spectral density $\sigma^{2}$, and $s(t)$ represents the received UWB ranging signal, which can be expressed by (1) by replacing $\tilde{\omega}(t)$ by $\omega(t)$ to reflect the effects of the antennas.

In this paper, a single-user scenario is considered, and $c_{j}=$ $0 \forall j$ is assumed for the rest of the paper. Then, the received signal can be expressed as

$$
r(t)=\sqrt{E_{\mathrm{s}}} \sum_{j=-\infty}^{\infty} \sum_{l=1}^{L} a_{j} \alpha_{l} \omega\left(t-\tau_{l}-j T_{\mathrm{f}}\right)+n(t) .
$$

\section{THEORETICAL LIMITS}

\section{A. $C R L B$}

The CRLB sets a lower bound on the mean-squared-error (MSE) of an unbiased estimator, and maximum likelihood (ML) estimators can achieve this bound asymptotically under certain conditions [4], [5]. For the received signal in (2) that is observed over an interval $t \in\left[0, T_{\mathrm{obs}}\right]$, with $T_{\mathrm{obs}}=N_{\mathrm{r}} T_{\mathrm{s}}$, the log-likelihood function of $\boldsymbol{\theta}=\left[\begin{array}{ll}\boldsymbol{\alpha} & \boldsymbol{\tau}\end{array}\right]$ is given by [4]

$$
\Lambda(\boldsymbol{\theta})=k-\frac{1}{2 \sigma^{2}} \int_{0}^{N_{\mathrm{r}} T_{\mathrm{s}}}\left|r(t)-\sum_{l=1}^{L} \alpha_{l} s\left(t-\tau_{l}\right)\right|^{2} d t
$$

where $k$ is a term that is independent of $\boldsymbol{\theta}$.

From (4), the Fisher information matrix (FIM) can be obtained as follows ${ }^{1}$ [3]

$$
\mathbf{I}_{\boldsymbol{\theta}}=\left[\begin{array}{ll}
\mathbf{I}_{\alpha \alpha} & \mathbf{I}_{\alpha \tau} \\
\mathbf{I}_{\alpha \tau}^{T} & \mathbf{I}_{\tau \tau}
\end{array}\right],
$$

where $\quad \mathbf{I}_{\alpha \alpha}=\kappa E_{\mathrm{p}} \operatorname{diag}\{1, \ldots, 1\}, \quad \mathbf{I}_{\alpha \tau}=$ $\kappa E_{\mathrm{p}}^{\prime} \operatorname{diag}\left\{\alpha_{1}, \ldots, \alpha_{L}\right\}, \quad \mathbf{I}_{\tau \tau}=\kappa E_{\mathrm{p}}^{\prime \prime} \operatorname{diag}\left\{\alpha_{1}^{2}, \ldots, \alpha_{L}^{2}\right\}$, $\kappa=\frac{N_{\mathrm{r}} E_{\mathrm{s}}}{\sigma^{2}}, E_{\mathrm{p}}^{\prime}=\int_{0}^{T_{\mathrm{p}}} \omega(t) \omega^{\prime}(t) d t$, and $E_{\mathrm{p}}^{\prime \prime}=\int_{0}^{T_{\mathrm{p}}}\left|\omega^{\prime}(t)\right|^{2} d t$,

${ }^{1}$ It is assumed that no inter-pulse interference occurs. 
with $E_{\mathrm{p}}$ denoting the energy of the received UWB pulse, and $\omega^{\prime}(t)$ denoting the first derivative of $\omega(t)$. From (5), the CRLB for the path delays $\tau_{l}$, for $l=1,2, \ldots, L$, can be calculated as [3]

$$
\begin{aligned}
\operatorname{CRLB}\left(\tau_{l}\right) & =\left[\left(\mathbf{I}_{\tau \tau}-\mathbf{I}_{\alpha \tau}^{T} \mathbf{I}_{\alpha \alpha}^{-1} \mathbf{I}_{\alpha \tau}\right)^{-1}\right]_{l, l} \\
& =\frac{\sigma^{2}}{N_{\mathrm{r}} E_{\mathrm{s}}\left(E_{\mathrm{p}}^{\prime \prime}-E_{\mathrm{p}}^{\prime 2} / E_{\mathrm{p}}\right) \alpha_{l}^{2}} .
\end{aligned}
$$

It is observed that the CRLB depends on the pulse shape, the path gains, and the signal-to-noise ratio (SNR).

\section{B. $Z Z L B$}

The CRLB can yield quite tight limits on time-delay ("range") estimation in high SNR scenarios. However, for moderate and low SNRs, the CRLB may result in a loose limit for MSEs of unbiased time-delay estimators. Unlike the CRLB, the ZZLB can provide tight limits for a wide range of SNRs. The ZZLB in an additive white Gaussian noise (AWGN) channel can be derived as [6]

$$
\text { ZZLB }=\frac{1}{T_{\mathrm{a}}} \int_{0}^{T_{\mathrm{a}}}\left(T_{\mathrm{a}}-\zeta\right) \zeta \mathrm{P}_{\mathrm{opt}}(\zeta) d \zeta,
$$

where $0<\tau_{1} \leq T_{\mathrm{a}}$, and $\mathrm{P}_{\text {opt }}$ is given by

$$
\mathrm{P}_{\mathrm{opt}}(\zeta)=Q\left(\sqrt{\frac{2 E_{\mathrm{p}}}{\sigma^{2}}\left(1-R_{\omega}(\zeta)\right)}\right),
$$

where $R_{\omega}(\zeta)$ denotes the auto-correlation function of the received pulse $\omega(t)$. The ZZLB can be evaluated also for multipath channels, as studied in [6]. Although the ZZLB cannot be obtained in closed forms in many cases, it provides a tighter bound than the CRLB for low-to-medium SNRs.

\section{ML BASEd RANGing TeChniques}

In this section, range estimation based on ML techniques is investigated [2]. Starting from the assumption of full $a$ priori information, various degrees of assumptions about the unknown parameters are considered [3], [7], [8]. In addition, the generalized ML ratio test approach and the sub-Nyquist sampling ML approaches are discussed [9], [10].

\section{A. ML Estimation with Full A-priori Information}

ML time-of-arrival (TOA) estimation (equivalently, ranging) can be performed by means of a correlator with a template signal that is perfectly matched to the received multipath signal, and by determining the time delay of the template for which the correlator output is maximized [3]. That optimal template can be expressed as

$$
s_{\mathrm{tmp}}(t)=\sum_{l=1}^{L} \alpha_{l} s\left(t-\tau_{l}\right) .
$$

However, this ML receiver cannot be implemented in practice, since the received waveform has unknown parameters to be estimated, i.e., delays, amplitudes, and pulse shapes of different multipath components (MPCs).

\section{B. ML Estimation with No Prior Information}

Although the main parameter of interest in TOA estimation is the delay of the first arriving signal path, $\tau_{1}$, the TOA estimation problem is closely related to the channel estimation problem, in which all the parameters of $\boldsymbol{\theta}=$ $\left[\begin{array}{lllllll}\tau_{1} & \cdots & \tau_{L} & \alpha_{1} & \cdots & \alpha_{L}\end{array}\right]$ are estimated [7], [8]. Let the samples of the received signal be denoted by $r[i]$ and the samples of the reconstructed signal be given by $\hat{r}[i]=\sum_{l=1}^{L} \hat{\alpha}_{l} \omega\left(i T_{\mathrm{smp}}-\hat{\tau}_{l}\right)$, where $T_{\mathrm{smp}}$ is the sampling interval. Then, for Gaussian noise, the ML solution is given by [7]

$$
\hat{\boldsymbol{\theta}}=\arg \min _{\boldsymbol{\theta}}\left\{\frac{1}{N_{\mathrm{smp}}} \sum_{i=1}^{N_{\mathrm{smp}}}|r[i]-\hat{r}[i]|^{2}\right\} .
$$

Then, the ML estimates for the elements of the unknown parameter vector $\boldsymbol{\theta}=\left[\begin{array}{ll}\boldsymbol{\tau} & \boldsymbol{\alpha}\end{array}\right]$ are given by [8]

$$
\begin{aligned}
& \hat{\boldsymbol{\tau}}=\arg \max _{\boldsymbol{\tau}}\left\{\left[\boldsymbol{\Omega}^{T}(\boldsymbol{\tau}) \mathbf{r}\right]^{T} \mathbf{R}_{\Omega}^{-1}(\boldsymbol{\tau})\left[\boldsymbol{\Omega}^{T}(\boldsymbol{\tau}) \mathbf{r}\right]\right\}, \\
& \hat{\boldsymbol{\alpha}}=\mathbf{R}_{\Omega}^{-1}(\hat{\boldsymbol{\tau}}) \boldsymbol{\Omega}^{T}(\boldsymbol{\tau}) \mathbf{r},
\end{aligned}
$$

where $\mathbf{R}_{\Omega}(\boldsymbol{\tau})=\boldsymbol{\Omega}^{T}(\boldsymbol{\tau}) \boldsymbol{\Omega}(\boldsymbol{\tau})$ with

$$
\begin{aligned}
& \boldsymbol{\Omega}^{T}(\boldsymbol{\tau})=\left[\boldsymbol{\omega}^{\left(D_{1}\right)} \boldsymbol{\omega}^{\left(D_{2}\right)} \cdots \boldsymbol{\omega}^{\left(D_{L}\right)}\right. \\
& \boldsymbol{\omega}^{\left(D_{l}\right)}=\left[\begin{array}{ll}
\mathbf{0}_{D_{l}} \boldsymbol{\omega} & \mathbf{0}_{N_{\mathrm{smp}}-N_{\boldsymbol{\omega}}-D_{l}}
\end{array}\right]^{T}[2] .
\end{aligned}
$$

Note that the ML estimation of the delays and the channel coefficients has high computational complexity as it requires a search over different values of $\tau$. For a resolvable channel, i.e., $\left|\tau_{i}-\tau_{j}\right| \geq T_{\mathrm{p}} \forall i \neq j$, the unknown parameter vector can be estimated as [7]

$$
\hat{\boldsymbol{\tau}}=\arg \max _{\boldsymbol{\tau}}\left\{\sum_{l=1}^{L} \frac{\left(\left[\boldsymbol{\omega}^{\left(D_{l}\right)}\right]^{T} \mathbf{r}\right)^{2}}{R_{\Omega}(0)}\right\}, \hat{\boldsymbol{\alpha}}=\frac{\boldsymbol{\Omega}^{T}(\hat{\boldsymbol{\tau}}) \mathbf{r}}{R_{\Omega}(0)},
$$

where $R_{\Omega}(0)=E_{\mathrm{p}}$. Note that for a resolvable channel, $\hat{\boldsymbol{\tau}}$ in (13) can be estimated by maximizing each term in the sum separately.

\section{Ranging with Generalized Maximum Likelihood Ratio Test}

The ML estimation based on the channel estimation problem provides an optimal TOA estimate. However, it is quite impractical for UWB channels with a large number of MPCs as the estimation of all the channel parameters is computationally very intensive. Therefore, a simplified version of the ML approach, called generalized maximum likelihood (GML) technique, is studied in [9]. Assuming that the strongest MPC has been detected, the GML technique estimates the TOA by performing a search over a smaller search space. Specifically, the paths prior to the strongest MPC are investigated.

Let $\tilde{r}(t)=r\left(t+\tau_{\text {peak }}\right) /\left|\alpha_{\text {peak }}\right|$ represent the normalized signal prior to and including the strongest MPC, where $\tau_{\text {peak }}$ and $\alpha_{\text {peak }}$ are the delay and the channel coefficient of the strongest MPC, respectively. Then, the samples of $\tilde{r}(t)$ at above the Nyquist rate can be expressed as [9]

$$
\tilde{\mathbf{r}}=\tilde{\alpha}_{1} \boldsymbol{\omega}_{\tilde{\tau}_{1}}+\sum_{l=2}^{L_{\max }} \tilde{\alpha}_{l} \boldsymbol{\omega}_{\tilde{\tau}_{l}}+\tilde{\mathbf{n}},
$$

where $\boldsymbol{\omega}_{\tilde{\tau}_{l}}$ denote the samples from $\omega\left(t+\tilde{\tau}_{l}\right), \tilde{\tau}_{l}=\tau_{\text {peak }}-\tau_{l}$, $\tilde{\alpha}_{l}=\alpha_{l} /\left|\alpha_{\text {peak }}\right|, L_{\max }$ is the number of MPCs prior to and including the strongest MPC, and $\tilde{\mathbf{n}}$ represents white Gaussian noise samples.

Based on (14), the GML estimate of $\tilde{\tau}_{1}$ is given by [9]

$$
\hat{\tilde{\tau}}_{1}=\arg \max _{\tilde{\tau}_{1}}\left[\min _{\tilde{\alpha}_{1}, L_{\max }, \tilde{\boldsymbol{\alpha}}, \tilde{\boldsymbol{\tau}}}\left\|\tilde{\mathbf{r}}-\tilde{\alpha}_{1} \boldsymbol{\omega}_{\tilde{\tau}_{1}}-\sum_{l=2}^{L_{\max }} \tilde{\alpha}_{l} \boldsymbol{\omega}_{\tilde{\tau}_{l}}\right\|^{2}\right],
$$

where $\tilde{\boldsymbol{\alpha}}=\left[\tilde{\alpha}_{2}, \ldots, \tilde{\alpha}_{L_{\max }}\right]$ and $\tilde{\boldsymbol{\tau}}=\left[\tilde{\tau}_{2}, \ldots, \tilde{\tau}_{L_{\max }}\right]$. The computational complexity of this optimization problem is quite high due to a search over the unknown parameter set $\left\{\tilde{\tau}_{1}, \tilde{\alpha}_{1}, L_{\max }, \tilde{\boldsymbol{\alpha}}, \tilde{\boldsymbol{\tau}}\right\}$. Therefore, an iterative technique with lower complexity is proposed in [9], which uses certain prior 
statistics of the UWB channel to define a new search space and to set a stopping rule.

\section{Sub-Nyquist Sampling ML Estimation with Various Levels of A-Priori Information}

The ML techniques discussed in Section IV-B and Section IV-C can be impractical in many cases due to their computational complexity and high sampling rate requirements. In this section, TOA estimators that operate at low sampling rates and with various levels of a-priori information are described.

Let $z[n]$ denote the samples of an energy detector output ${ }^{2}$ for $n=1,2, \ldots, N_{\mathrm{b}}$. It is assumed that the samples indexed by $n=k, \ldots, k+N_{\mathrm{e}}-1$ include both the desired signal and noise, whereas the remaining samples are only due to the noise. Note that each possible value of $k$ represents a different TOA, and can be considered as one of the hypotheses in a hypothesis testing problem. Let $\mathbf{z}_{k}^{(\mathrm{sn})}\left(\mathbf{z}_{k}^{(\mathrm{no})}\right)$ represent a row vector of size $N_{\mathrm{e}}\left(N_{\mathrm{b}}-N_{\mathrm{e}}\right)$ that consists of the samples from the signal-plus-noise (noise-only) part.

a) Maximum Energy Selection: The maximum energy selection (MES) approach is a simple technique that estimates the TOA according to the strongest sample, i.e.,

$$
\hat{n}_{\text {toa }}^{\text {(mes) }}=\arg \max _{k \in\left\{1, \ldots, N_{\mathrm{b}}\right\}}\{z[k]\} .
$$

In typical UWB channels, $N_{\mathrm{e}}$ can be much larger than 1 when the sampling interval is on the order of a pulse duration. Therefore, there can be a large delay between the first path and the strongest path, and the MES technique can yield large estimation errors.

b) Maximum Energy Sum Selection: In order to obtain more reliable TOA estimates, the energy of adjacent samples can be combined. According to the maximum energy sum selection (MESS) technique with a window duration of $N_{\mathrm{w}} \leq$ $N_{\mathrm{e}}$ samples, the leading edge estimate is given by

$$
\hat{n}_{\text {toa }}^{\text {(mess) }}=\arg \max _{k \in\left\{1, \ldots, N_{\mathrm{b}}\right\}}\left\{\mathbf{z}_{k}^{\left(\mathrm{sn}, N_{\mathrm{w}}\right)} \mathbf{1}_{N_{\mathrm{w}}}\right\},
$$

where $\mathbf{z}_{k}^{\left(\mathrm{sn}, N_{\mathrm{w}}\right)}$ is composed of the first $N_{\mathrm{w}}$ elements of $\mathbf{z}_{k}^{(\mathrm{sn})}$ and $\mathbf{1}_{N_{\mathrm{w}}}$ is an $N_{\mathrm{w}} \times 1$ column vector of ones. Note that MESS reduces to MES for $N_{\mathrm{w}}=1$. Since a large window can capture a large amount of noise and a small window may not capture sufficient signal energy, there exists an optimal window length that depends on the channel realization and the SNR.

c) Weighted Maximum Energy Sum Selection: In the presence of a-priori knowledge about the channel power delay profile, the weighted-MESS (W-MESS) technique can be used:

$$
\hat{n}_{\text {toa }}^{\text {(wmess) }}=\arg \max _{k \in\left\{1, \ldots, N_{\mathrm{b}}\right\}}\left\{\mathbf{z}_{k}^{\left(\mathrm{sn}, N_{\mathrm{e}}\right)} \boldsymbol{\rho}_{N_{\mathrm{e}}}\right\},
$$

where $\boldsymbol{\rho}_{N}$ is a column vector of size $N_{\mathrm{e}}$ that provides weighting of different samples. For example, $\rho_{N_{\mathrm{e}}}$ can consist of mean energy values for a particular channel model and block duration in an energy detector implementation. In addition, if the channel energies are perfectly known, the TOA estimate can be obtained from

$$
\hat{n}_{\text {toa }}^{(\text {wmess') }}=\arg \max _{k \in\left\{1, \ldots, N_{\mathrm{b}}\right\}}\left\{\mathbf{z}_{k}^{\left(\mathrm{sn}, N_{\mathrm{e}}\right)} \mathcal{E}_{N_{\mathrm{e}}}\right\} .
$$

d) Double-Weighted Maximum Energy Sum Selection: At the correct timing value, the mean $\hat{\mu}_{k}^{(\text {no) }}$ and the standard deviation $\hat{\sigma}_{k}^{(\text {no) }}$ of $\mathbf{z}_{k}^{(\text {no) }}$ are minimized. Therefore, weighing

\footnotetext{
${ }^{2}$ Similarly, the absolute values of correlation outputs can be considered
}

the energy sum in (17) by the inverse of $\hat{\mu}_{k}^{(\mathrm{no})} \hat{\sigma}_{k}^{(\mathrm{no})}$ will increase the likelihood of the correct hypothesis. This estimator is called double-weighted MESS (DW-MESS) and is given by

$$
\hat{n}_{\text {toa }}^{\text {(dw-mess) }}=\arg \max _{k \in\left\{1, \ldots, N_{\mathrm{b}}\right\}}\left\{\frac{\mathbf{z}_{k}^{\left(\mathrm{sn}, N_{\mathrm{e}}\right)} \boldsymbol{\rho}_{N_{\mathrm{e}}}}{\hat{\mu}_{k}^{(\mathrm{no})} \hat{\sigma}_{k}^{(\text {(no) }}}\right\} .
$$

In addition to the MES, MESS, W-MESS and DW-MESS estimators, [10] also proposes a Bayesian estimator for energy detector based implementations, which requires prior information related to the statistics of individual energy detector samples. However, such prior information may not be available in practice.

\section{LOW COMPLEXITY RANGING TECHNIQUES}

In this section, various peak detection algorithms [7], twostep TOA estimation approaches [11], ranging with dirty templates [12], [13], and threshold based ranging algorithms [14]-[15] are discussed.

\section{A. Ranging with Largest-N Peak-Detection Techniques}

The MES technique in (15) may not yield an accurate TOA (range) estimate in the presence of MPCs prior to the strongest one. In order to improve the accuracy of range estimation, [7] proposes to consider the largest $N$ correlation peaks, and to select the time delay corresponding to the peak with the smallest time index.

Let the time indices corresponding to the MPCs with the largest $N$ correlation peaks be represented by $k_{1}, k_{2}, \ldots, k_{N}$, where $k_{i}$ represents the time index for the $i$ th strongest component. Then, the TOA of the received signal can be estimated as [7]

$$
\hat{\tau}_{1}=T_{\text {smp }} \min \left\{k_{1}, k_{2}, \ldots, k_{N}\right\}
$$

where $T_{\mathrm{smp}}$ denotes the sampling period of the receiver. In [7], the authors also propose two improvements for this technique in non-resolvable channels.

\section{B. Ranging with Two-Step TOA Estimators}

It is of significant importance to perform accurate range estimation without employing high sampling rates in UWB ranging systems. In order to have low-power and low-complexity implementations, symbol-rate or frame-rate samples should be considered [3], which can, however, increase the time to perform TOA estimation considerably. One technique to perform reasonably accurate range estimation based on lowrate samples is to employ two-step TOA estimators [11]. In order to perform TOA estimation in short time intervals, the first step of the two-step TOA estimator in [11] performs a coarse timing estimate using energy detection (similarly, the dirty template approach can be used). Then, the second step refines the TOA estimate based on a statistical change detection algorithm.

\section{Ranging with Dirty Templates}

Another low complexity TOA estimator is the dirty-template technique introduced in [12], [13], which operates on symbolrate samples. The main idea behind this technique is to use the received signal itself as a "dirty" template and then to perform cross-correlations with the symbol-length portions of the received signal. The main advantage of the dirty-template technique is its low complexity; however, the estimates obtained by this technique can have an ambiguity equal to the extent of the noise-only region between consecutive symbols. 


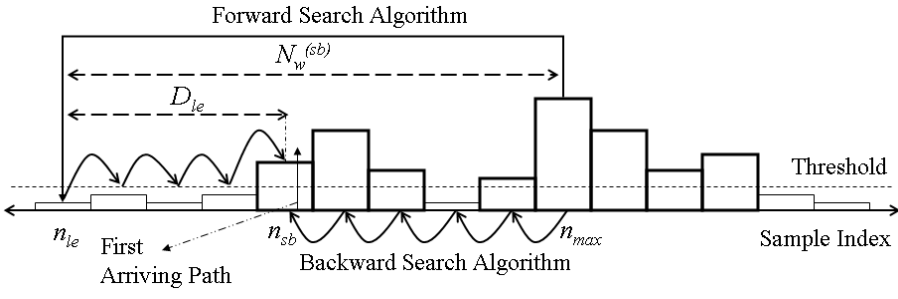

Fig. 1. "Jump back and search forward" (JBSF) and "serial backward search" (SBS) algorithms [2]. Here, $N_{\mathrm{w}}^{(\mathrm{sb})}$ denotes the search-back window length in samples, $n_{\max }$ is the index of the strongest sample, $n_{\text {le }}$ is the index of the first arriving path's sample, $n_{\mathrm{sb}}$ is index of the the first sample within the search-back window, $D_{\max }$ is the delay between the first arriving path's sample and the strongest sample, and $D_{\text {le }}$ is the delay between the index of the first sample within the search window and the first arriving path's sample.

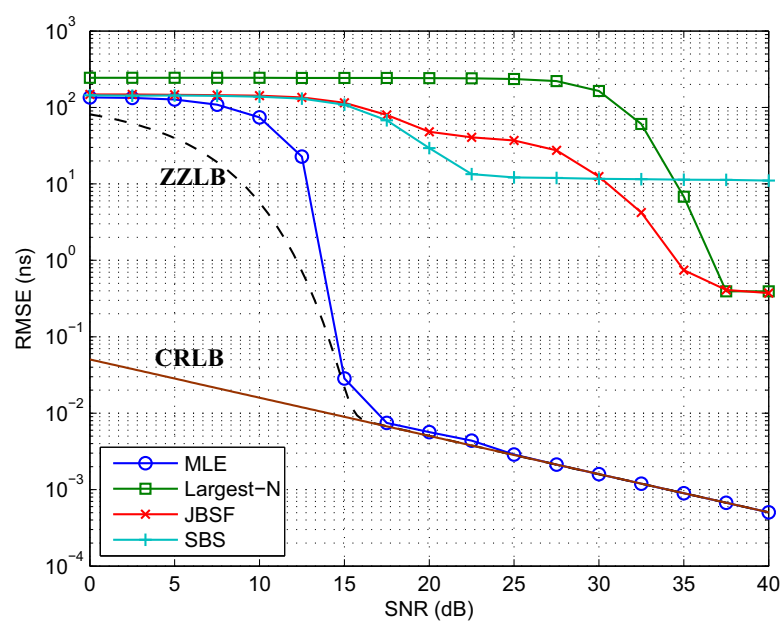

Fig. 2. RMSE vs. SNR for various algorithms, and the CRLB and the ZZLB.

\section{Threshold Based Ranging}

Threshold based ranging algorithms compare the received signal samples against a threshold in order to identify the first arriving MPC [14]. In [15], a simple threshold based algorithm is introduced, which compares absolute values of the direct samples of the received signal (or, samples of the correlator/energy detector output) against a threshold. The TOA of the signal is determined by the index of the first sample that exceeds the threshold. Since the signal energy and the channel impulse response are unknown, the threshold can be determined based only on the noise floor.

In [16], two different threshold based TOA estimation algorithms are proposed, which are summarized in Fig. 1. The thresholds of those algorithms can be set based on the noise level, or, using a normalized threshold as in [17]. In either case, certain statistics of the UWB channels can be used to select parameters for the search space and stopping rules accurately.

\section{Simulations ANd CONCluding REMARKS}

In this section, comparison of some of the TOA estimation (ranging) algorithms is presented together with the theoretical lower bounds. An uncertainty region of $500 \mathrm{~ns}$ is considered for TOA estimation, and 100 realizations from the CM-1 of the IEEE 802.15.4a channel model are employed [18]. A time resolution of $0.01 \mathrm{~ns}$ is considered, and the second derivative of the Gaussian pulse [2] with around $1 \mathrm{~ns}$ pulse width is used in the training signal (only one pulse is employed).

Fig. 2 illustrates the root-mean-squared-error (RMSE) values versus SNR for four different algorithms, namely, the ML estimator (MLE) in Section IV-A (considered as a benchmark), the largest- $N$ peak-detection algorithm in Section V-A, and the JBSF and the SBS techniques in Section V-D. ${ }^{3}$ The last three algorithms are considered due to their practicality. In addition, the ZZLB and the CRLB are illustrated. In Fig. 2, the MLE has the best performance as expected, and it gets very close to the ZZLB for almost all SNR values and to the CRLB only at high SNRs. Although the MLE has very good performance, it is impractical in most cases. Considering the practical estimators, the JBSF algorithm seems to have better performance than the other ones (in fact, the SBS has lower RMSEs for a certain SNR range), and the JBSF and the largest- $N$ peak-detection algorithms can provide sub-nanosecond accuracy. ${ }^{4}$

Consideration of theoretical limits together with practical algorithms provide an evaluation of how accurately TOA estimation can be performed under practical constraints. Further research is required to perform detailed analysis of various UWB ranging algorithms.

\section{REFERENCES}

[1] H. Arslan, Z. N. Chen, and M.-G. Di Benedetto, (editors) Ultra Wideband Wireless Communications, Wiley-Interscience, Oct. 2006.

[2] Z. Sahinoglu, S. Gezici, and I. Guvenc, Ultra-Wideband Positioning Systems: Theoretical Limits, Ranging Algorithms, and Protocols, Cambridge University Press, 2008.

[3] S. Gezici, Z. Tian, G. B. Giannakis, H. Kobayashi, A. F. Molisch, H. V. Poor and Z. Sahinoglu, "Localization via ultra-wideband radios," IEEE Signal Processing Magazine, vol. 22, issue 4, pp. 70-84, July 2005.

[4] H. V. Poor, An Introduction to Signal Detection and Estimation, New York: Springer-Verlag, 1994.

[5] E. Weinstein and A. J. Weiss, "Fundamental limitations in passive time delay estimation - Part II: Wide-band systems," IEEE Trans. Acoust. Speech, Sig. Processing, vol. 32, no. 5, pp. 1064-1078, Oct. 1984.

[6] D. Dardari, C. C. Chong, and M. Z. Win, "Improved lower bounds on time-of-arrival estimation error in realistic UWB channels," Proc. IEEE Int. Conf. on UWB (ICUWB), Waltham, MA, Sep. 2006, pp. 531-537.

[7] C. Falsi, D. Dardari, L. Mucchi, and M. Z. Win, "Time of arrival estimation for UWB localizers in realistic environments," EURASIP J. Applied Sig. Processing, pp. 1-13, 2006.

[8] M. Z. Win and R. A. Scholtz, "Characterization of ultrawide bandwidth wireless indoor channels: A communication theoretic view," IEEE J. Select. Areas. Commun., vol. 20, no. 9, pp. 1613-1627, Dec. 2002.

[9] J.-Y. Lee and R. A. Scholtz, "Ranging in a dense multipath environment using an UWB radio link," IEEE J. Select. Areas Commun., vol. 20, no. 9, pp. 1677-1683, Dec. 2002.

[10] I. Guvenc and Z. Sahinoglu, "TOA estimation with different IR-UWB transceiver types," Proc. IEEE Int. Conf. UWB, Zurich, Switzerland, Sep. 2005, pp. 426-431.

[11] S. Gezici, Z. Sahinoglu, A. F. Molisch, H. Kobayashi, and H. V. Poor, "Two-step time of arrival estimation for pulse based ultra-wideband systems," EURASIP Journal on Advances in Signal Processing, vol. 2008, Article ID 529134, 11 pages, 2008

[12] L. Yang and G. B. Giannakis, "Ultra-wideband communications: An idea whose time has come," IEEE Sig. Processing Mag., vol. 21, no. 6, pp. 26-54, Nov. 2004.

[13] L. Yang and G. B. Giannakis, "Timing ultra-wideband signals with dirty templates," IEEE Trans. Commun., vol. 53, no. 11, pp. 1952-1963, Nov. 2005 .

[14] D. Dardari and M. Z. Win, "Threshold-based time-of-arrival estimators in UWB dense multipath channels," Proc. IEEE Int. Conf. Commun. (ICC), vol. 10, Istanbul, Turkey, June 2006, pp. 4723-4728.

[15] R. A. Scholtz and J. Y. Lee, "Problems in modeling UWB channels," Proc. IEEE Asilomar Conf. Signals, Syst. Computers, vol. 1, Pacific Grove, CA, Nov. 2002, pp. 706-711.

[16] I. Guvenc, Z. Sahinoglu, A. F. Molisch, and P. Orlik, "Non-coherent TOA estimation in IR-UWB systems with different signal waveforms,", Proc. IEEE Int. Workshop on Ultrawideband Networks (UWBNETS), Boston, MA, October 2005, pp. 245-251.

[17] I. Guvenc and Z. Sahinoglu, "Threshold-based TOA estimation for impulse radio UWB systems," Proc. IEEE Int. Conf. UWB, Zurich, Switzerland, Sep. 2005, pp. 420-425.

[18] A. F. Molisch et. al., "IEEE 802.15.4a Channel Model - Final Report," http://www.ieee802.org/15/pub/TG4a.html, Sep. 2004.

${ }^{3}$ The thresholds for the JBSF and the SBS techniques are set to 0.25 times the maximum correlation output (the window size is $50 \mathrm{~ns}$ ), and $N=500$ for the largest- $N$ peak-detection algorithm.

${ }^{4}$ Note that the high SNR values in the figure may be obtained by means of pulse combining (only one pulse is considered in the simulations) 